\title{
Validation of the Korean Depressive Symptom Inventory-Suicidality Subscale
}

\author{
Sooyeon Suh ${ }^{1,2} \bowtie$, Hyera Ryu ${ }^{1}$, Carol $\mathrm{Chu}^{3}$, Melanie $\mathrm{Hom}^{3}$, Megan L. Rogers ${ }^{3}$, and Thomas Joiner ${ }^{3}$ \\ ${ }^{1}$ Department of Psychology, Sungshin Women's University, Seoul, Republic of Korea \\ ${ }^{2}$ Department of Psychiatry, Stanford University, Palo Alto, CA, USA \\ ${ }^{3}$ Department of Psychology, Florida State University, Tallahassee, FL, USA
}

\begin{abstract}
Objective The present study aimed to validate a Korean version of the self-report Depressive Symptom Inventory-Suicidality Subscale (DSI-SS).

Methods 553 South Korean undergraduate students (aged 18-34 years, 74.8\% females) completed questionnaires. Participants completed Korean versions of the self-report Depressive Symptom Inventory-Suicidality Subscale (DSI-SS), Beck Depression Inventory-II (BDI-II), Insomnia Severity Index (ISI), Beck Scale for Suicide Ideation (BSS), and a measure of suicide-related symptom history.

Results Results indicated that the DSI-SS demonstrated excellent internal consistency $(\alpha=0.93)$ and strong convergent validity with the BDI $(r=0.57, p<0.01)$, ISI $(r=0.27, p<0.01)$, and BSS $(r=0.70, p<0.01)$. DSI-SS scores also significantly differentiated between those reporting a history of suicide attempts compared to non-suicide attempters [t $(38.80)=-3.28, \mathrm{p}<0.05]$.

Conclusion Given the brevity of this measure, and evidence for its validity, the Korean version of the DSI-SS may be particularly promising for clinical and empirical use as a screening tool among South Korean undergraduates.
\end{abstract}

Psychiatry Investig 2017;14(5):577-584

Key Words Suicidal ideation, Suicide attempted, Korea, Validation studies, Risk assessment.

\section{INTRODUCTION}

Suicide is a leading causes of death worldwide ${ }^{1}$ and a particularly significant public health problem in South Korea. Suicide rates in South Korea are not only the highest among countries belonging to the Organization for Economic Cooperation and Development, ${ }^{2}$ but they have also markedly increased in the last decade from 10.8 to 29.1 per 100,000 people. ${ }^{2}$ Consequently, there is a clear need for studies that contribute to suicide prevention efforts in South Korea, including the development of brief screening measures that can be used to detect suicide risk.

The assessment of suicidal ideation and plans for suicide is

Received: June 29, 2016 Revised: August 18, 2016

Accepted: September 26, 2016 Available online: June 16, 2017

$\triangle$ Correspondence: Sooyeon Suh, $\mathrm{PhD}$

Department of Psychology, Sungshin Women's University, 2 Bomun-ro 34dagil, Seongbuk-gu, Seoul 02844, Republic of Korea

Tel: +82-2-920-7215, Fax: +82-2-920-2040, E-mail: alysuh@sungshin.ac.kr

(a) This is an Open Access article distributed under the terms of the Creative Commons Attribution Non-Commercial License (http://creativecommons.org/licenses/bync/4.0) which permits unrestricted non-commercial use, distribution, and reproduction in any medium, provided the original work is properly cited. one of the most common methods used to screen for suicide risk. ${ }^{3,4}$ The utility of assessing for suicidal ideation has been supported by previous studies, as individuals who experience severe suicidal ideation are more likely to have a history of multiple suicide attempts.-7 Additionally, other studies have shown that approximately $34 \%$ of individuals aged $15-54$ years transitioned from suicidal ideation to a plan within 1-2 years. ${ }^{7,8}$ This pattern of findings has also been found in South Korea, with individuals who experienced suicidal ideation having a 30 times higher risk of suicide attempts than those who had not experienced ideation. ${ }^{9,10}$ Given the prevalence of suicidal ideation among South Korean university students, ${ }^{11}$ there is a critical need for brief, effective Korean-language tools that can be used to routinely screen for suicidal ideation in university, clinical, and medical settings. ${ }^{12}$

In Korea, there are several suicide risk assessment tools that have been validated [e.g., Beck Scale for Suicide Ideation (BSS), Reasons For Living Inventory (RFL)], ${ }^{13,14}$ but these scales are typically lengthy. In addition, several scales which have shown good reliability and validity in detecting suicidal ideation [e.g., Adult Suicidal Ideation Questionnaire (ASIQ), Risk As- 
sessment Suicidality Scale (RASS)] have not been validated in Korean samples. ${ }^{15,16}$ The only brief Korean language screening tool that has been validated for use in Korea is a two-item measure developed by Kim et al. ${ }^{17}$ However, this screening measure is clinician-administered and not validated for use as a self-report measure. Thus, it may be difficult to implement in clinical settings with limited resources. Additionally, the items in Kim and colleagues ${ }^{17}$ measure were selected by seven psychiatrists who utilized subjective criteria for item selection rather than empirical approaches (e.g., factor analysis). Further, this measure has only been validated for use in older adults. There is a need for a validated, suicide-related symptom screening tool, particularly for use among Korean young adults, ${ }^{17}$ because of higher risk in this population.

The four-item Depressive Symptom Inventory-Suicidality Subscale (DSI-SS; ${ }^{18-20}$ has been validated in Australian and North American samples, and thus may also be a useful measure of suicidality in Korean individuals. In a study of Australian youths (aged 15-24 years), the DSI-SS which is brief and easily administered exhibited good reliability and validity. ${ }^{18}$ As expected, DSI-SS assessed suicidality was significantly related more severe depressive symptoms, and general emotional distress. ${ }^{18}$ Moreover, the authors suggested a cut-off total score of 3 or above as an optimal score for predicting clinically significant suicide risk. Recently, Batterham and colleagues ${ }^{19}$ conducted a systematic review of measures of suicidality and found that the DSI-SS was a particularly useful measure for the general population due to its superior psychometric properties. Given the utility of the DSI-SS in other populations, we sought to empirically establish the validity of a Korean-language version of the DSI-SS in a sample of Korean undergraduate students in the present study.

To investigate convergent validity of the Korean version of DSI-SS, we examined the relationship between the DSI-SS and measures of depression and insomnia symptoms. Multiple studies have established that depression is a strong risk factor for suicidal ideation, ${ }^{21-24}$ with suicidal ideation consistently significantly associated with greater depression symptom severity, ${ }^{25}$ Additionally, insomnia has been shown to be an independent risk factor for suicidal ideation, with more severe insomnia symptoms predicting more severe suicidal ideation. ${ }^{26-30}$ That sleep disturbances are listed among the top 10 warning signs of suicide by the Substance Abuse and Mental Health Services Administration (SAMHSA) emphasizes the importance of assessing sleep disturbance during suicide risk screening. ${ }^{31}$ Insomnia and depression have also both been shown to be significantly associated with suicidal ideation in Korea, specifically. ${ }^{11,32,33}$ Thus, we examined correlations between the Korean DSI-SS and measures of depression and insomnia to evaluate its convergent validity.
In the current study, we investigated the internal reliability, factor structure, and convergent and discriminant validity of the Korean DSI-SS as a suicide risk screening tool among South Korean undergraduate students. We collected comprehensive information regarding previous suicide attempts to investigate whether the Korean DSI-SS would be correlated with past suicide attempt history. We hypothesized that the Korean DSI-SS would demonstrate strong psychometric properties, similar to results yielded by similar studies conducted with the English version of the DSI-SS in other countries, and that it would also be significantly associated with depression, insomnia, and another measure of suicidal ideation.

\section{METHODS}

\section{Participants}

A sample of 554 undergraduate students was recruited from four universities in Seoul and Daejeon, Korea. The sample consisted of 412 females (74.8\%) and 139 males (25.2\%), aged $18-34$ years (Mage $=21.87$ years, $S D=2.23$ ). Data for one participant were excluded due to missing DSI-SS data; thus, 553 participants were included in our final analyses. The mean age of participants was $21.87 \pm 2.23$ years (range: $18-34$ years), with $74.5 \%$ of the sample being female $(n=412)$. The most common living arrangements were living with parents (53.4\%), followed by living alone (25\%), living with friends (11.3\%), others $(8.3 \%)$, living with relatives $(1.3 \%)$, or a partner $(0.7 \%)$.

\section{Procedures and measures}

All participants provided informed consent prior to study participation and were compensated with course credit after completion of the questionnaire battery. This study was approved by the Institutional Review Board of each university where data collection took place. First, the DSI-SS was translated from English into Korean by a bilingual individual with prior translation experience who was independent from the study. After translation, the Korean DSI-SS was back-translated to English by a second bilingual individual. This backtranslated version was then compared with the original English version of the DSI-SS. Finally, further modifications (e.g., word choice to enhance clarity) were made to yield the final version of the Korean DSI-SS used for this study.

\section{Demographic information}

Information regarding age, gender, living arrangements, satisfaction with university life, and current perceived health were provided by students on a self-report survey.

\section{Depressive Symptom Inventory-Suicidality Subscale}

The DSI-SS is a subscale of the Hopelessness Depression 
Symptom Questionnaire. It was developed as a brief screening tool for suicide risk in general health settings. The DSI-SS consists of 4 items assessing the frequency and intensity of suicidal ideation, formulation of plans for suicide, controllability of suicidal thoughts, and suicide-related impulses during the past 2 weeks. Each item is rated on a scale from 0 to 3 (total score range $0-12$ ), with higher scores reflecting greater severity of suicidal ideation. The internal consistency in this sample was excellent (Cronbach's alpha=0.93).

\section{Beck Depression Inventory-II}

The BDI-II is a self-report inventory used to assess the severity of depressive symptoms during the past two weeks. ${ }^{34}$ It consists of 21 items that assess cognitive, emotional, physical, and motivational symptoms of depression. Each item is rated on a $0-3$ scale (total score range: $0-63$ ), with higher scores reflecting greater severity of depressive symptoms. The BDIII has been previously validated in a Korean sample and Cronbach's alpha was $0.88 .{ }^{35}$ The internal consistency of the BDIII in this sample was excellent (Cronbach's alpha=0.90).

\section{Insomnia Severity Index}

The ISI is a 7-item self-report inventory developed by Bastien, Vallieres, and Morin ${ }^{36}$ that assesses severity of insomnia symptoms during past two weeks. Each item is rated on a 5-point likert scale ranging from 0 to 4 , with higher scores reflecting greater severity of insomnia symptoms (total score range: 0 to 28). Items assess the severity of various insomnia symptoms, including satisfaction with sleep and degree of worry about sleep problems. The ISI has previously been validated in Korean samples and Cronbach's alpha was $0.92 .{ }^{37}$ The internal consistency in this sample was adequate (Cronbach's alpha=0.76).

\section{Beck Scale for Suicide Ideation}

The BSS is a 19-item self-report inventory developed by Beck, Steer and Ranieri. ${ }^{38}$ The BSS assesses the severity of suicidal thoughts and associated symptoms, such as the extent of an individual's wish to die, desire to make an actual suicide attempt, and subjective feelings of control over one's thoughts of suicide. Each item is rated on a 3-point scale ranging from 0 to 2 (total score range $0-38$ ). A Korean-language version of the BSS has previously been validated in a Korean sample and Cronbach's alpha was $0.74 .^{13}$ The internal consistency in this sample was good (Cronbach's alpha=0.88).

\section{Suicide attempt history}

All participants answered comprehensive questions regarding their history of suicidal behaviors. Specifically, participants were asked whether they had a history of suicide at- tempts, and if relevant, they were asked to provide the following information regarding prior attempts: number, intention (e.g., "I didn't want to die, I just needed help"), methods (e.g., drugs, chemicals, asphyxia, jumping, self-inflicting wound), and whom they informed before or after the suicide attempt. Participants were also asked whether, to their knowledge, any of their family members and/or friends had a suicide attempt history.

\section{Statistical analyses}

Inter-correlations and corrected item-total correlations of the Korean DSI-SS were analyzed by Spearman correlation analyses. Cronbach's alpha was used to quantify the internal consistency of the Korean DSI-SS. To establish convergent validity, a Pearson correlation analysis was conducted between scores on the DSI-SS, BDI-II, ISI and BSS. Additionally, multiple linear regression analyses were conducted to determine whether DSI-SS scores predicted BSS scores after controlling for BDI-II and ISI scores. Finally, t-tests were computed between suicide attempters and non-attempters to verify whether the DSI-SS was useful in discriminating between attempters and non-attempters.

\section{RESULTS}

\section{Clinical characteristics}

In this study, the mean total score for self-report measures were as follows: DSI-SS $=1.03 \pm 1.85$ (range $0-11$ ), BDI-II $=9.16 \pm$ 7.66 (range $0-41$ ), ISI $=8.46 \pm 4.64$ (range $0-25$ ), and $\mathrm{BSS}=$ $4.51 \pm 5.14$ (range $0-29)$. In this sample, $8.3 \%(n=46)$ of participants met criteria for clinical depression (BDI $\geq 22)$ and 9.9\% ( $n=55)$ met criteria for clinical levels of insomnia (ISI $\geq 15)$ based on empirically derived cut-off scores from Korean samples. ${ }^{35,39}$ Demographic and clinical characteristics of the sample can be found in Table 1 .

\section{Inter-item characteristics and internal consistency of the suicide screen}

Inter-item correlations and corrected item-total correlations of the four items from the DSI-SS are presented on the diagonal in Table 2. All inter-item correlations were at least moderate $(>0.60)$. The corrected item-total correlation ranged from 0.75 to 0.88 . These correlations were high and indicated that the 4 items consist of a coherent, one-dimensional scale. The Cronbach's alpha for the Korean DSI-SS was 0.93, indicating the four items of the suicide measure had excellent internal consistency.

In addition to inter-item correlations, factor analysis using principal axis factoring was conducted with the four DSI-SS items. One large factor emerged, with the eigenvalue for the 
Table 1. Demographic information and suicide attempt history $(\mathrm{N}=553)$

\begin{tabular}{|c|c|}
\hline Demographic information & $\mathrm{N}(\%)$ or mean $(\mathrm{SD})$ \\
\hline \multicolumn{2}{|l|}{ Gender } \\
\hline Male & $139(25.1)$ \\
\hline Female & $412(74.5)$ \\
\hline Missing & $2(0.4)$ \\
\hline Age & $21.87(2.23)$ \\
\hline \multicolumn{2}{|l|}{ Living arrangement } \\
\hline Alone & $138(24.9)$ \\
\hline Spouse/significant other & $4(0.7)$ \\
\hline Parents & $294(53.2)$ \\
\hline Friends & $62(11.2)$ \\
\hline Relatives & $7(1.3)$ \\
\hline Others & $46(8.3)$ \\
\hline Missing & $2(0.4)$ \\
\hline Suicide attempt history & $\mathrm{N}(\%)$ \\
\hline \multicolumn{2}{|l|}{ 1. Previous suicide attempt } \\
\hline Yes & $38(6.9)$ \\
\hline No & $511(93.1)$ \\
\hline \multicolumn{2}{|l|}{ 1-1. Number of suicide attempts } \\
\hline 1 & $21(65.6)$ \\
\hline 2 & $6(18.8)$ \\
\hline 3 & $5(15.6)$ \\
\hline \multicolumn{2}{|l|}{ 2. Suicide intention $(\mathrm{N}=31)$} \\
\hline \multicolumn{2}{|c|}{$\begin{array}{l}\text { a) I really wanted to die, and the only reason I didn't die is because } \\
\text { of luck }\end{array}$} \\
\hline Yes & $8(25.8)$ \\
\hline No & $23(74.2)$ \\
\hline
\end{tabular}

b) I tried killing myself, but I knew the method I chose wasn't perfect

Yes $25(80.6)$

No $6(19.4)$

c) I didn't want to die, I just needed help

Yes $17(54.8)$

No 14 (45.2)

3. Method of suicide attempt*

1) Drug overdose

Yes $10(26.3)$

No $22(57.9)$

Missing 6(15.8)

Yes $10(26.3)$

No $22(57.9)$

Missing 6(15.8)

2) Chemicals

Yes $\quad 0(0)$

No $\quad 31(81.6)$
Table 1. Demographic information and suicide attempt history $(\mathrm{N}=553)$ (continued)

\begin{tabular}{lr}
\hline \multicolumn{1}{c}{ Suicide attempt history } & $\mathrm{N}(\%)$ \\
\hline Missing & $7(18.4)$ \\
3) Asphyxia & $6(15.8)$ \\
Yes & $26(68.4)$ \\
No & $6(15.8)$ \\
Missing & \\
4) Jumping down & $9(23.7)$ \\
Yes & $24(63.2)$ \\
No & $5(13.2)$ \\
Missing & \\
5) Self-inflicting wound & $20(52.6)$ \\
Yes & $11(28.9)$ \\
No & $7(18.4)$ \\
Missing
\end{tabular}

*participants were allowed to endorse multiple methods of suicide attempts

first factor (3.29) being significantly larger than the eigenvalue for the second factor (0.35). All four items loaded strongly on one factor (all loadings $>0.60$ ), providing evidence for a cohesive, internally consistent scale.

\section{Convergent validity of DSI-SS}

To assess convergent validity of the DSI-SS, DSI-SS suicidal symptoms were correlated with BDI-II depression, ISI insomnia, and BSS suicidal symptoms. The correlation coefficient between the DSI-SS and BDI-II was 0.577 ( $p<0.01$ ), 0.279 with the ISI $(\mathrm{p}<0.01)$, and 0.706 with the BSS $(\mathrm{p}<0.01)$. Additionally, we examined whether the DSI-SS was correlated with the BSS after controlling for depression and insomnia symptoms. Linear regression analyses indicated that DSI-SS scores remained a significant predictor of BSS scores $(\beta=0.57, \mathrm{p}<0.001)$, even after controlling for depression $(\beta=0.24, \mathrm{p}<0.001)$ and insomnia ( $\beta=-0.86, p=0.01)$ symptoms. The results of this study support the convergent validity of DSI-SS. Table 3 presents correlations of DSI-SS, BDI-II, ISI and BSS scores.

\section{Distribution of DSI-SS in a general sample of young adults}

Cut-off scores are often used in clinical settings to inform risk level determinations and guide treatment. Table 4 provides the frequency distributions and descriptive statistics for all participants. The DSI-SS mean was $1.03(\mathrm{SD}=1.85)$ in the overall sample. This DSI-SS mean score was higher than that found in a previous study of adolescents and young adults in Australia (M=0.79, $\mathrm{SD}=1.65) .{ }^{18}$ The cut-off score suggested for use in Joiner and colleagues' original study ${ }^{18}$ was 3, which corresponded to approximately $15 \%$ of the sample. In our sample, 
$15.7 \%$ of the sample corresponded to a cut-off score of 4 . The corresponding total scores were 15.68 for the BDI-II and 9.68 for the ISI, which are markedly elevated and in the clinical range.

\section{Discriminant validity in differentiating attempters vs. non-attempters}

Differences between suicide attempters and non-suicide attempters can be found in Table 5. Group differences in age, gender, current perceived health, university life satisfaction, DSI-SS, ISI, BDI-II, and BSS total scores were examined. Although there was no significant association between number of previous suicide attempts and DSI-SS scores among participants with a history of suicide attempts $(r=0.06, p=0.73)$, There was a significant difference in DSI-SS scores between suicide attempters and non-suicide attempters $[\mathrm{t}(38.80)=-3.28$, $\mathrm{p}<0.05]$. Thus, DSI-SS demonstrates promise in being able to differentiate between suicide attempters and non-suicide at-

Table 2. Inter-correlations of Individual Items of the Depressive Symptom Inventory-Suicidality Subscale

\begin{tabular}{lccccc}
\hline & Item 1 & Item 2 & Item 3 & Item 4 \\
\hline Item 1 (suicidal ideationfrequency) & 0.88 & & & \\
Item 2 (plans for an attempt) & 0.83 & 0.84 & & \\
Item 3 (suicidal ideation intensity) & 0.84 & 0.80 & 0.87 & \\
Item 4 (impulse to kill oneself) & 0.73 & 0.67 & 0.72 & 0.75 \\
\hline
\end{tabular}

Inter-correlations of individual items are below the diagonal; corrected item-total correlations are on the diagonal

Table 3. Associations between depression symptoms, insomnia symptoms, and suicide ideation

\begin{tabular}{lllll}
\hline & DSI-SS & BDI-II & ISI & BSS \\
\hline DSI-SS & 1 & & & \\
BDI-II & $0.57^{* *}$ & 1 & & \\
ISI & $0.27^{* *}$ & $0.42^{* *}$ & 1 & \\
BSS & $0.70^{* *}$ & $0.55^{* *}$ & $0.16^{* *}$ & 1 \\
\hline
\end{tabular}

${ }^{* *} \mathrm{p}<0.01$. DSI-SS: Depressive Symptom Inventory-Suicidality Subscale, BDI-II: Beck Depression Inventory, ISI: Insomnia Severity Index, BSS: Beck Scale for Suicidal Ideation tempters, providing evidence for its discriminant validity.

\section{DISCUSSION}

Suicide is a growing public health concern in South Korea 2 , particularly among university students 11 , yet, to our knowledge, no Korean-language suicide screening measures have been validated for use among Korean young adults. Thus, the purpose of the current study was to evaluate the psychometric properties of the Korean DSI-SS in a sample of South Korean college students.

Importantly, in this study, the Korean DSI-SS demonstrated excellent internal consistency ( $\alpha=0.93)$, which suggests that the items on the Korean DSI-SS are related but not redundant. This finding is in line with previous studies in undergraduate samples that have found that the original English

Table 4. Distribution of Depressive Symptom Inventory-Suicidality subscale Scores

\begin{tabular}{cccc}
\hline DSI-SS score & $\mathrm{N}(\%)$ & BDI-II, mean (SD) & ISI, mean (SD) \\
\hline 0 & $388(70)$ & $6.61(5.50)$ & $7.76(4.36)$ \\
1 & $28(5.1)$ & $12.57(7.02)$ & $9.07(3.60)$ \\
2 & $16(2.9)$ & $11.69(7.28)$ & $8.07(5.74)$ \\
3 & $35(6.3)$ & $11.54(6.41)$ & $10.14(4.02)$ \\
4 & $57(10.3)$ & $15.68(7.88)$ & $9.68(5.37)$ \\
5 & $15(2.7)$ & $23.20(8.81)$ & $11.53(4.49)$ \\
6 & $5(0.9)$ & $21.40(7.13)$ & $13.60(2.51)$ \\
7 & $6(1.1)$ & $26.17(10.15)$ & $14.83(4.88)$ \\
8 & $1(0.2)$ & $15.00(-)$ & $9.00(-)$ \\
9 & - & - & - \\
10 & - & - & - \\
11 & $2(0.4)$ & $36.00(-)$ & $22.00(-)$ \\
12 & - & - & - \\
Total & $553(100)$ & $9.18(7.66)$ & $8.45(4.64)$ \\
\hline
\end{tabular}

DSI-SS: Depressive Symptom Inventory-Suicidality Subscale, BDIII: Beck Depression Inventory, ISI: Insomnia Severity Index

Table 5. Differences between suicide attempters vs. non-attempters

\begin{tabular}{lccc}
\hline \multicolumn{1}{c}{ Variables } & Suicide attempters [mean (SD) or N (\%)] & Non-suicide attempters [mean $(\mathrm{SD})$ or N (\%)] & p-value \\
\hline Age & $21.88(2.24)$ & $21.51(2.05)$ & 0.343 \\
Gender & $29(76.3 \%)$ female & $381(74.7 \%)$ female & 0.501 \\
Current health & $2.92(1.10)$ & $2.44(0.93)$ & $0.003^{*}$ \\
University life satisfaction & $2.53(0.76)$ & $2.40(0.68)$ & 0.285 \\
DSI-SS & $2.53(2.98)$ & $0.92(1.69)$ & $0.002^{*}$ \\
ISI & $9.43(5.28)$ & $8.39(4.59)$ & 0.199 \\
BDI-II & $15.13(10.46)$ & $8.76(7.23)$ & $0.001^{*}$ \\
BSS & $11.37(8.28)$ & $4.02(4.45)$ & $<0.001^{* *}$ \\
\hline
\end{tabular}

${ }^{*} \mathrm{p}<0.05,{ }^{* *} \mathrm{p}<0.001$. DSI-SS: Depressive Symptom Inventory-Suicide subscale, ISI: Insomnia Severity Index, BDI-II: Beck Depression Inventory-II, BSS: Beck Scale for Suicidal Ideation 
version of the DSI-SS exhibits favorable internal consistency (e.g., $\alpha s=0.80^{40}$ and $0.90,{ }^{18}$ respectively). The strong reliability of this measure supports the use of the Korean DSI-SS among Korean college student populations.

In addition to reliability, we also evaluated the validity of the Korean DSI-SS. In terms of the measure's convergent validity, we found that the Korean DSI-SS was strongly correlated with an empirically established Korean measure of suicidal ideation (i.e., BSS).$^{13}$ This indicates that our measure is capturing a construct highly related to suicidal ideation. Further, consistent with study hypotheses, the Korean DSI-SS was moderately correlated with depression symptoms and less strongly but significantly related to insomnia severity. This is consistent with prior research indicating that depression is one of the most robust predictors of suicidal ideation and behavior. ${ }^{21}$ These results also align with findings from previous research studies reporting similar correlations between the English DSI-SS and validated measures of depression $(\mathrm{r}=0.60)^{18}$ and insomnia $(\mathrm{r}=0.21)^{41}$ symptoms. Taken together, these results appear to support the convergent validity of the Korean DSI-SS.

We also sought to evaluate the discriminant validity of the Korean DSI-SS with respect to suicide attempt history. Our results revealed that the Korean DSI-SS distinguished between individuals with and without a history of suicide attempts, such that individuals with a history of suicide attempts reported significantly more severe symptoms on the Korean DSI-SS. Attempt history was similarly distinguishable on the basis of the Korean BSS. This finding further strengthens the validity of the Korean DSI-SS. These results are also congruent with prior research showing that individuals with a history of suicide attempts are at greater risk for subsequent suicidal ideation following an attempt. ${ }^{42}$ Overall, the results from this study indicate that the Korean DSI-SS is likely a reliable and valid measure of suicidal ideation when used in a Korean undergraduate population.

Finally, in many clinical settings, it is desirable to establish cut-off scores to guide diagnosis and treatment. While it would have been ideal to utilize more sophisticated statistical methods, such as Receiver Operating Curve (ROC) analysis, to establish cut-off scores, the lack of an empirically derived cut-off score for the alternate suicide measure that we used in the current study (i.e., the Korean-language version of the BSS) challenged our ability to conduct these analyses. Thus, we utilized frequency distributions proposed by the original authors. ${ }^{18}$ Our evidence suggests that a cut-off score of 4 may be more appropriate than the English DSI-SS recommended cut-off score of $3 .^{18}$ This higher cut-off score for the Korean DSI-SS may reflect cultural differences both in the experiencing and reporting of suicide risk. For instance, lower levels of suicidal ideation may not be experienced as similarly distressing or clinically significant among Korean undergraduates as compared to those from Western cultures. Korean undergraduates-especially those with less severe ideation-may also more willingly disclose ideation. Replication of these results in other clinically well-characterized samples will be necessary to test these conjectures and better understand this discrepancy.

Identification of this cut-point also has important implications for suicide prevention efforts in Korea. For one, a cut-off score may be used to distinguish high and low suicide risk groups within the context of suicide prevention research in South Korea-an endeavor that is particularly important given the high rates of suicide in this country. ${ }^{2}$ Additionally, the DSISS's cut score may be useful in clinical settings as a screening measure for suicide risk. The brevity, reliability, and validity of the Korean DSI-SS are also ideal for clinical use. However, given that this study has only established cut-off scores in Korean undergraduate student samples, caution is warranted when applying this cut-score to other populations.

\section{Limitations and future directions}

Although these preliminary findings are promising, the study was also limited in several ways. First, as mentioned previously, given that we only collected data among two South Korean university samples, findings may not be generalizable to other university populations or sociodemographic groups in South Korea (e.g., non-college attending young adults, other age groups, mental health inpatients and outpatients). Thus, further work is needed to examine the psychometric properties of the Korean DSI-SS across additional samples, especially since such research would inform whether the DSI-SS may be useful as a population-wide suicide risk screening measure, as is the case with the original English-language version of the DSI-SS. ${ }^{19}$

Second, we were limited in the constructs that we were able to utilize to determine convergent validity (i.e., only suicidal ideation, depression, and insomnia). There is immense clinical utility in being able to detect short-term risk among individuals at elevated risk for suicide; thus, future research seeking to replicate our findings would likely benefit from inclusion of measures examining acute suicide risk factors (e.g., agita$\operatorname{tion}^{43,44}$ ). Third, due to our cross-sectional study design, we were unable to examine the predictive validity of the Korean DSI-SS. Again, since we ultimately aim to utilize this measure to identify South Korean individuals at elevated risk for engaging in suicidal behaviors (e.g., lethal and near-lethal attempts), empirical testing of the predictive validity of this measure is critical. Thus, we look forward to future research that employs a prospective approach.

Finally, sole reliance on self-report measures in assessing 
convergent validity represented another limitation of this study since it may have led to common method variance. Future studies validating the Korean DSI-SS would be strengthened by an examination of the convergence between this measure and clinician-assessed ideation and/or implicitly assessed ideation. ${ }^{45}$ At the same time, however, it is important to note that the Korean DSI-SS is not intended to replace other risk suicide assessment procedures. Instead, this brief, selfreport measure is primarily intended for use in suicide risk screening such that individuals with clinically significant suicide risk can be referred for further evaluation and care. Thus, it is not imperative that the DSI-SS demonstrate perfect alignment with these other assessment modalities.

In conclusion, this study aimed to establish the psychometric properties of the Korean-language version of the DSISS in a sample of South Korean university students. Findings revealed that the Korean DSI-SS demonstrated good internal consistency and convergent validity with suicide-related symptoms measures. Although further work is needed to replicate our present findings across samples and to establish the measure's ability to predict suicidal behaviors over time, given the brief and readily administered nature of this measure, the Korean DSI-SS appears particularly promising for clinical and empirical use as a screening tool among undergraduates in South Korea.

\section{Acknowledgments}

This work was supported by the Sungshin University Research Grant of 2016 (2016-1-29-006).

\section{REFERENCES}

1. WHO. Injuries and violence: the facts. Switzerland: World Health Organization; 2014.

2. OECD. Health at a Glance 2015: OECD Indicators. Paris: OECD Publishing; 2015.

3. Chu C, Klein KM, Buchman-Schmitt JM, Hom MA, Hagan CR Joiner TE. Routinized assessment of suicide risk in clinical practice: an empirically informed update. J Clin Psychol 2015;71:1186-1200.

4. Linehan MM, Comtois KA, Ward-Ciesielski EF. Assessing and managing risk with suicidal individuals. Cogn Behav Pract 2012;19:218-232.

5. Brent DA, Perper JA, Moritz G, Allman C, Friend A, Roth C, et al. Psychiatric risk factors for adolescent suicide: a case-control study. J Am Acad Child Adolesc Psychiatry 1993;32:521-529.

6. Nock MK, Borges G, Bromet EJ, Alonso J, Angermeyer M, Beautrais A, et al. Cross-national prevalence and risk factors for suicidal ideation, plans and attempts. Br J Psychiatry 2008;192:98-105.

7. Kessler RC, Borges G, Walters EE. Prevalence of and risk factors for lifetime suicide attempts in the National Comorbidity Survey. Arch Gen Psychiatry 1999;56:617-626.

8. Nock MK, Banaji MR. Prediction of suicide ideation and attempts among adolescents using a brief performance-based test. J Consult Clin Psychol 2007;75:707-715.

9. Park E. The influencing factors on suicide attempt among adolescents in South Korea. J Korean Acad Nurs 2008;38:465-473.

10. Cho SJ, Jeon HJ, Kim JK, Suh TW, Kim SU, Hahm BJ, et al. Prevalence of suicide behaviors (suicidal ideation and suicide attempt) and risk factors of suicide attempts in junior and high school adolescents. J Korean Neuropsychiatr Assoc 2002;41:1142-1155.

11. Jeon HJ, Lee JY, Lee YM, Hong JP, Won SH, Cho SJ, et al. Lifetime prevalence and correlates of suicidal ideation, plan, and single and multiple attempts in a Korean nationwide study. J Nerv Ment Dis 2010;198:643646.

12. Schmitz WM Jr, Allen MH, Feldman BN, Gutin NJ, Jahn DR, Kleespies PM, et al. Preventing suicide through improved training in suicide risk assessment and care: an American Association of Suicidology Task Force report addressing serious gaps in US mental health training. Suicide Life Threat Behav 2012;42:292-304.

13. Lee H, Kwon J. Validation for the Beck scale for suicide ideation with Korean university students. Korean J Clin Psychol 2009;28:1155-1172.

14. Lee G, Hyun M, Lee S. A study on the validation of the reasons for living inventory. Korean J Health Psychol 2010;15:227-242.

15. Reynolds WM. Psychometric characteristics of the Adult Suicidal Ideation Questionnaire in college students. J Pers Assess 1991;56:289-307.

16. Fountoulakis KN, Pantoula E, Siamouli M, Moutou K, Gonda X, Rihmer Z, et al. Development of the Risk Assessment Suicidality Scale (RASS): a population-based study. J Affect Disord 2012;138:449-457.

17. Kim J, Kang E, Jeong JW, Paik JW. Korean suicide risk screening tool and its validity. J Kor Cont Assoc 2013;13:240-250.

18. Joiner TE Jr, Pfaff JJ, Acres JG. A brief screening tool for suicidal symptoms in adolescents and young adults in general health settings: reliability and validity data from the Australian National General Practice Youth Suicide Prevention Project. Behav Res Ther 2002;40:471-481.

19. Batterham PJ, Ftanou M, Pirkis J, Brewer JL, Mackinnon AJ, Beautrais A, et al. A systematic review and evaluation of measures for suicidal ideation and behaviors in population-based research. Psychol Assess 2015;27:501-512.

20. Metalsky GI, Joiner TE Jr. The hopelessness depression symptom questionnaire. Cogn Ther Res 1997;21:359-384.

21. Nock MK, Hwang I, Sampson NA, Kessler RC. Mental disorders, comorbidity and suicidal behavior: results from the National Comorbidity Survey Replication. Mol Psychiatry 2010;15:868-876.

22. Brown GK, Beck AT, Steer RA, Grisham JR. Risk factors for suicide in psychiatric outpatients: a 20-year prospective study. J Consult Clin Psychol 2000;68:371-377.

23. Malone KM, Haas GL, Sweeney JA, Mann JJ. Major depression and the risk of attempted suicide. J Affect Disord 1995;34:173-185.

24. May AM, Klonsky ED. What distinguishes suicide attempters from suicide ideators? A meta-analysis of potential factors. Clin Psychol Sci Pract 2016;23:5-20.

25. Hawton K, Casañas I, Comabella C, Haw C, Saunders K. Risk factors for suicide in individuals with depression: a systematic review. J Affect Disord 2013;147:17-28.

26. Bernert RA, Joiner TE, Cukrowicz KC, Schmidt NB, Krakow B. Suicidality and sleep disturbances. Sleep 2005;28:1135-1141.

27. Ribeiro JD, Pease JL, Gutierrez PM, Silva C, Bernert RA, Rudd MD, et al. Sleep problems outperform depression and hopelessness as crosssectional and longitudinal predictors of suicidal ideation and behavior in young adults in the military. J Affect Disord 2012;136:743-750.

28. Goldstein TR, Bridge JA, Brent DA. Sleep disturbance preceding completed suicide in adolescents. J Consult Clin Psychol 2008;76:84-91.

29. Bernert RA, Turvey CL, Conwell Y, Joiner TE Jr. Association of poor subjective sleep quality with risk for death by suicide during a 10-year period: a longitudinal, population-based study of late life. JAMA Psychiatry 2014;71:1129-1137.

30. McCall WV, Blocker JN, D’Agostino R, Kimball J, Boggs N, Lasater B, et al. Insomnia severity is an indicator of suicidal ideation during a depression clinical trial. Sleep Med 2010;11:822-827.

31. Pigeon WR, Britton PC, Ilgen MA, Chapman B, Conner KR. Sleep disturbance preceding suicide among veterans. Am J Pub Health 2012; 102(Suppl 1):S93-S97.

32. Lee HS, Kim S, Choi I, Lee KU. Prevalence and risk factors associated 
with suicide ideation and attempts in Korean college students. Psychiatry Investig 2008;5:86-93.

33. Suh S, Kim H, Yang HC, Cho ER, Lee SK, Shin C. Longitudinal course of depression scores with and without insomnia in non-depressed individuals: a 6-year follow-up longitudinal study in a Korean cohort. Sleep 2013;36:369-376.

34. Beck AT, Steer RA, Brown GK. Beck Depression Inventory-II. San Antonio, TX: Psychological Corporation; 1996.

35. Sung H, Kim J, Park Y, Bai D, Lee S, Ahn H. A study on the reliability and the validity of Korean version of the Beck Depression Inventory-II (BDI-II). J Korean Soc Biol Ther Psychiatry 2008;14:201-212.

36. Bastien $\mathrm{CH}$, Vallieres A, Morin CM. Validation of the Insomnia Severity Index as an outcome measure for insomnia research. Sleep Med 2001;2:297-307.

37. Cho YW, Song ML, Morin CM. Validation of a Korean Version of the Insomnia Severity Index. J Clin Neurol 2014;10:210-215.

38. Beck AT, Steer RA, Ranieri WF. Scale for suicide ideation: psychometric properties of a self-report version. J Clin Psychol 1988;44:499-505.

39. Cho YW, Song ML, Morin CM. Validation of a Korean version of the insomnia severity index. J Clin Neurol 2014;10:210-215.

40. Chu C, Buchman-Schmitt JM, Moberg FB, Joiner TE. Thwarted belongingness mediates the relationship between fear of negative evaluation and suicidal ideation. Cognit Ther Res 2016;40:31-37.

41. Cukrowicz KC, Otamendi A, Pinto JV, Bernert RA, Krakow B Joiner Jr TE. The impact of insomnia and sleep disturbances on depression and suicidality. Dreaming 2006;16:1-10.

42. Vilhjálmsson R, Kristjansdottir G, Sveinbjarnardottir E. Factors associated with suicide ideation in adults. Soc Psychiatry Psychiatr Epidemiol 1998;33:97-103.

43. Fawcett J, Scheftner WA, Fogg L, Clark DC, Young MA, Hedeker D, et al. Time-related predictors of suicide in major affective disorder. Am J Psychiatry 1990;147:1189-1194.

44. Hall RC, Platt DE, Hall RC. Suicide risk assessment: a review of risk factors for suicide in 100 patients who made severe suicide attempts: evaluation of suicide risk in a time of managed care. Psychosomatics 1999;40:18-27.

45. Nock MK, Banaji MR. Assessment of self-injurious thoughts using a behavioral test. Am J Psychiatry 2007;164:820-823. 